\title{
Inconsistency in Abnormal Brain Activity across Cohorts of ADHD-200 in Children with Attention Deficit Hyperactivity Disorder
}

\author{
Jian-Bao Wang 1,2,3, Li-Jun Zheng 1, 2,3, Qing-Jiu Cao ${ }^{4}$, Yu-Feng Wang ${ }^{4}$, Li Sun ${ }^{4}$, \\ Yu-Feng Zang ${ }^{1,2,3 *}$ and Hang Zhang ${ }^{5 *}$ \\ ${ }^{1}$ Center for Cognition and Brain Disorders and the Affiliated Hospital, Hangzhou Normal University, Hangzhou, China, \\ ${ }^{2}$ Zhejiang Key Laboratory for Research in Assessment of Cognitive Impairments, Hangzhou, China, ${ }^{3}$ Institutes of \\ Psychological Sciences, College of Education, Hangzhou Normal University, Hangzhou, China, ${ }^{4}$ Institute of Mental Health, \\ The Sixth Hospital, Peking University, Beijing, China, ${ }^{5}$ Paul C. Lauterbur Research Centers for Biomedical Imaging, Shenzhen \\ Institutes of Advanced Technology, Chinese Academy of Sciences, Shenzhen, China
}

\section{OPEN ACCESS}

Edited by:

Bharat B. Biswal,

University of Medicine and Dentistry of New Jersey, United States

Reviewed by:

Mitul Ashok Mehta,

King's College London, United

Kingdom

Mingrui Xia,

Beijing Normal University, China

*Correspondence:

Yu-Feng Zang

zangyf@gmail.com

Hang Zhang

kevinhangbnu@foxmail.com

Specialty section: This article was submitted to

Brain Imaging Methods,

a section of the journal

Frontiers in Neuroscience

Received: 11 January 2017 Accepted: 19 May 2017

Published: 06 June 2017

Citation:

Wang J-B, Zheng L-J, Cao Q-J, Wang $Y-F$, Sun $L$, Zang $Y-F$ and

Zhang $H$ (2017) Inconsistency in

Abnormal Brain Activity across

Cohorts of ADHD-200 in Children with

Attention Deficit Hyperactivity

Disorder. Front. Neurosci. 11:320.

doi: 10.3389/fnins.2017.00320
Many papers have shown results from the multi-site dataset of resting-state fMRI (rs-fMRI) in attention deficit hyperactivity disorder (ADHD), a data-sharing project named ADHD-200. However, few studies have illustrated that to what extent the pooled findings were consistent across cohorts. The present study analyzed three voxel-wise whole-brain metrics, i.e., amplitude of low-frequency fluctuation (ALFF), regional homogeneity (ReHo), and degree centrality (DC) based on the pooled dataset as well as individual cohort of ADHD-200. In addition to the conventional frequency band of $0.01-0.08 \mathrm{~Hz}$, sub-frequency bands of 0-0.01, 0.01-0.027, 0.027-0.073, 0.073-0.198, and 0.198$0.25 \mathrm{~Hz}$, were assessed. While the pooled dataset showed abnormal activity in some brain regions, e.g., the bilateral sensorimotor cortices, bilateral cerebellum, and the bilateral lingual gyrus, these results were highly inconsistent across cohorts, even across the three cohorts from the same research center. The standardized effect size was rather small. These findings suggested a high heterogeneity of spontaneous brain activity in ADHD. Future studies based on multi-site large-sample dataset should be performed on pooled data and single cohort data, respectively and the effect size must be shown.

Keywords: attention deficit hyperactivity disorder, resting state fMRI, multi-site dataset, ADHD-200, voxel-wise whole-brain analysis

\section{INTRODUCTION}

Attention deficit hyperactivity disorder (ADHD) is one of the most common neurodevelopmental disorders in children (Polanczyk et al., 2015). It is a highly heterogeneous disease, involving multiple deficits and multiple neural pathways (Castellanos et al., 2006; Bush, 2010). The complicated pathophysiology of ADHD has been widely investigated through task and restingstate functional magnetic resonance imaging (fMRI) studies. Task-state fMRI studies commonly employed various task paradigms, e.g., Go/No Go (Schulz et al., 2004; Newman et al., 2015), Eriksen Flanker Task (Vaidya et al., 2005; Vasic et al., 2014). These tasks are complicated, and various paradigms did not exhibit consistent results (Cortese et al., 2012). In contrast, resting-state fMRI (rs-fMRI) is easy to be implemented and provides a consistent approach for clinical investigations. Thus, more and more researchers perform rs-fMRI studies on brain disorders, including ADHD. 
ADHD-200, as one of the most widely used multi-site MRI dataset of brain disorders, has attracted considerable attention from the ADHD research community. This dataset released by ADHD-200 consortium contains ten independent cohorts from eight different sites (ADHD-200-Consortium, 2012). These cohorts provide rs-fMRI and anatomical MRI data of both ADHD and typically developing children (TDC), about 776 participants in total. ADHD-200 facilitated the investigation of the neural basis of ADHD, and about 30 studies based on this dataset have been published according to PubMed (e.g., Tomasi and Volkow, 2012; Elton et al., 2014; Sripada et al., 2014; Carmona et al., 2015).

Most studies on ADHD-200 pooled data of cohorts and explored the abnormal brain activity for ADHD. Increasing number of these studies were reported in recent years. For example, Mills et al. (2012) pooled data of Brown University (BU), Peking University (PKU), Kennedy Krieger Institute (KKI), and New York University (NYU) together and observed increased connection between the medial and anterior dorsal thalamus and the basal ganglia in ADHD (Mills et al., 2012). Pooling data of PKU, NYU together, Zhang et al. (2014) found affected brain regions in ADHD mainly located in the orbitofrontal cortex, inferior/superior frontal gyrus, anterior cingulate gyrus, and calcarine cortex (Zhang et al., 2014). Pooling cohorts together facilitated the establishment of a large sample size and tended to provide very positive results. However, to what extent the pooled results are consistent across individual cohorts remains unknown. To the best of our knowledge, only one study on ADHD-200 dataset answered this question (Cai et al., 2015). They found that ADHD group of cohorts NYU, PKU, and OHSU consistently showed decreased network-interaction among the salience network (SN), central executive network (CEN), and default mode network (DMN). Notably, the network analysis could not indicate the exact aberrant brain regions for ADHD, and it remains unclear whether findings of the local brain regions for ADHD are consistent across cohorts or not.

The present study aimed to examine the consistency of abnormal local brain regions across cohorts of ADHD-200. Specifically, we analyzed three voxel-wise whole-brain metrics, i.e., amplitude of low-frequency fluctuation (ALFF) (Zang et al., 2007), regional homogeneity (ReHo) (Zang et al., 2004), and degree centrality (DC) (Buckner et al., 2009). Importantly, the analytic processes of these kinds of methods are very similar across studies, and hence facilitate the coordinate-based metaanalysis (CB-meta) which helps to find regions of consistent activity across fMRI studies (Bartra et al., 2013; Herz et al., 2014; Iwabuchi et al., 2015). Analysis of these metrics is often performed at the frequency band of $0.01-0.08 \mathrm{~Hz}$ which has been widely used in rs-fMRI studies. In addition to this conventional band, rs-fMRI signals at some sub-frequency bands can also be modulated by different resting state (e.g., eyes closed and eyes open; Yuan et al., 2014) as well as by disease (e.g., chronic pain; Malinen et al., 2010; Otti et al., 2013). These sub-frequency bands, i.e., Slow-6 (<0.01 Hz; Lv et al., 2013; Zhang et al., 2015a), Slow-5 (0.01-0.027 Hz), Slow-4 (0.027-0.073 Hz; Zuo et al., 2010; Han et al., 2011; Zhang et al., 2013), Slow-3 (0.073-0.198 Hz), and Slow-2 (0.198-0.25 Hz; Wang et al., 2015), were also investigated in the present study in order to obtain more information through the frequency-dependent characteristic.

\section{METHODS AND MATERIALS}

\section{Subjects and Data Acquisition}

The data we used in this study is publicly available from the ADHD-200 Consortium (http://fcon_1000.projects.nitrc. org/indi/adhd200/). The ADHD-200 dataset contains both functional and anatomical MRI data contributed by eight institutions. Each cohort was approved by the research ethics review boards of each institution. Signed informed consent was obtained from all participants or their legal guardian before participation.

We first selected the data cohorts according to the following criteria: (1) Including both $\mathrm{ADHD}$ and TDC groups. So the data from the BU, University of Pittsburgh and, Washington University were excluded; (2) Employing the same TR with $<2,000 \mathrm{~ms}$ across the cohort. According to this criterions, data from NeuroImage (TR $=1,960 \mathrm{~ms})$, KKI $(\mathrm{TR}=2,500 \mathrm{~ms})$, and OHSU (TR $=2,500 \mathrm{~ms}$ ) were excluded. Then, the NYU, PKU1, PKU2, and PKU3 cohorts were included in our research. The PKU2 and PKU3 cohorts only had male subjects, so the female subjects in NYU and PKU1 cohorts were excluded to remove potential confounding effect of gender to the consistency across cohort. Left-handedness subjects were also excluded for each cohort. After case-by-case matching age between ADHD and TDC, 58 subjects from NYU, 30 from PKU1, 56 from PKU2, and 38 from PKU3 were included in the current study. Demographic information was summarized in Table 1. Flow-chart of data exclusion was shown in Figure 1.

Psychostimulant medications were withheld at least $24 \mathrm{~h}$ prior to scanning. The inclusion and exclusion criteria and more detailed demographic characteristics of the participants of the four cohorts can be seen in the http://fcon_1000.projects.nitrc. org/indi/adhd200/. The rs-fMRI data of the four cohorts were from three scanners, with TR of $2 \mathrm{~s}$ for all. PKU1 and PKU2 used the same scanner but scanning parameters were slightly different. The detailed parameters were listed in the Supplementary Table 1.

\section{Data Preprocessing}

Functional images of each subject were preprocessed by using Data Processing Assistant for Resting-State fMRI (DPARSF) (Chao-Gan and Yu-Feng, 2010) which is based on Statistical Parametric Mapping (SPM8) (http://www.fil.ion.ucl.ac.uk/spm) and Resting-State fMRI Data Analysis Toolkit (Song et al., 2011). Preprocessing was performed as follows: removal of the first ten volumes to avoid signal instability and to get subjects adapted to the scanning noise. Then, the number of time point is 170 at least (NYU), so the first 170 volumes were included for individuals in PKU1, PKU2, and PKU3 considering the comparability across cohorts (Molloy et al., 2014; Carmona et al., 2015). Slice timing correction, image realignment to correct head motion were followed. After individual structural images were segmented after co-registered to functional images, functional images were spatial normalized to Montreal Neurological Institute (MNI) space at 3 $\mathrm{mm}$ isotropic voxel resolution applying the unified segmentation 
TABLE 1 | Demographic information of each cohort in the current study.

\begin{tabular}{|c|c|c|c|c|c|c|c|c|}
\hline & \multicolumn{2}{|c|}{ NYU } & \multicolumn{2}{|c|}{ PKU1 } & \multicolumn{2}{|c|}{ PKU2 } & \multicolumn{2}{|c|}{ PKU3 } \\
\hline & ADHD & TDC & ADHD & TDC & ADHD & TDC & ADHD & TDC \\
\hline$N$ & 29 & 29 & 15 & 15 & 28 & 28 & 19 & 19 \\
\hline Gender (male) & 29 & 29 & 15 & 15 & 28 & 28 & 19 & 19 \\
\hline Age (years) & $12.1 \pm 2.9$ & $12.2 \pm 2.8$ & $11.2 \pm 2.3$ & $11.6 \pm 1.5$ & $12.7 \pm 1.7$ & $11.7 \pm 1.8$ & $13.2 \pm 1.3$ & $13.3 \pm 1.0$ \\
\hline$I Q$ & $106.1 \pm 16.0$ & $115.3 \pm 14.3$ & $101.7 \pm 12.4$ & $123.0 \pm 14.2$ & $111.5 \pm 12.7$ & $121.6 \pm 12.2$ & $102.7 \pm 10.4$ & $111.7 \pm 12.7$ \\
\hline Subtype (C/l/H) & 19/10/0 & - & $9 / 6 / 0$ & - & $16 / 12 / 0$ & - & $12 / 7 / 0$ & - \\
\hline
\end{tabular}

Data are presented as mean $\pm S D$. C, $A D H D$-Combined; I, ADHD -Inattentive; H, ADHD -Hyperactive/Impulsive.

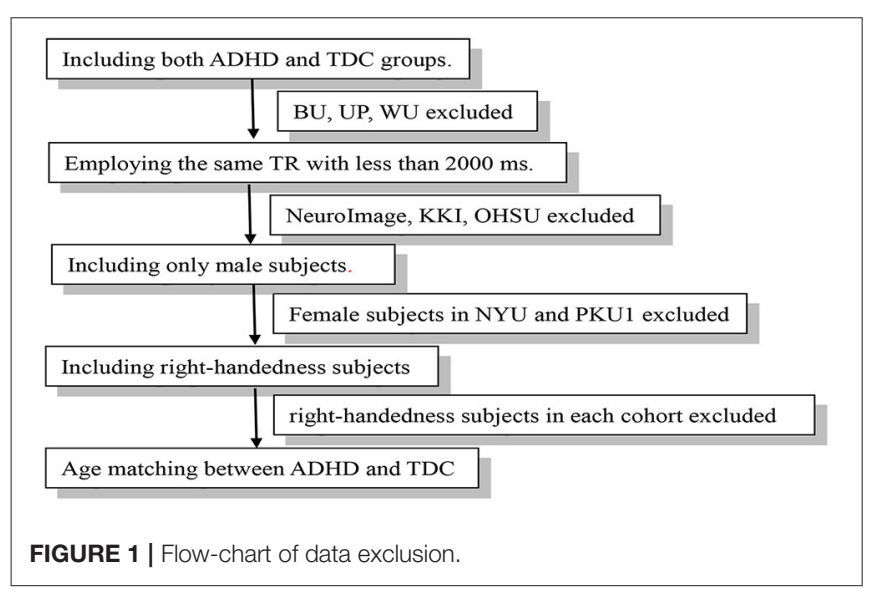

parameters. The linear trend, head motion parameter measured by Friston-24 model, white matter (WM), and cerebrospinal fluid (CSF) signals were further regressed out as nuisance covariates. Then, three voxel-wise whole-brain analytic methods, i.e., ALFF, $\mathrm{ReHo}$, and DC, were further used to analyze these preprocessed data.

\section{ALFF Calculation}

ALFF is the amplitude of low frequency fluctuations of the blood oxygen level dependent (BOLD) signal of every single voxel (Zuo et al., 2010). ALFF calculation was the same as the procedure in Zang et al. (2007). After preprocessing, the 4D rs-fMRI data of each participant was spatially smoothed with a $6 \mathrm{~mm}$ FWHM Gaussian kernel and then, the linear trend was removed from the time course of each voxel. Then, ALFF was calculated for the conventional low frequency band $(0.01-0.08 \mathrm{~Hz})$ as well as five sub-bands, i.e., Slow-6 $(0-0.01 \mathrm{~Hz})$, Slow-5 $(0.01-0.027 \mathrm{~Hz})$, Slow-4 (0.027-0.073 Hz), Slow-3 (0.073-0.198 Hz), and Slow-2 $(0.198-0.25 \mathrm{~Hz})$.

\section{ReHo Calculation}

ReHo is a voxel-wise measure of the local synchronization of the time courses of nearest neighboring voxels (usually 27 voxels). It was calculated by using Kendall's coefficient of concordance (KCC) as follows:

$$
W=\frac{\sum\left(R_{i}\right)^{2}-n(\bar{R})^{2}}{\frac{1}{12} K^{2}\left(n^{2}-n\right)}
$$

where $\mathrm{W}$ is the KCC among given voxels, ranged from 0 to 1 ; $R_{i}$ is the sum rank of the $i$ th time point; $\bar{R}=((n+1) K / 2)$ is the mean of $R_{i}$ 's; $K$ is the number of time courses within a measured cluster (27 in the current study); and $n$ is the number of ranks. After the removing of linear trend, the time course of each voxel, band-pass filtering was performed for six sub-bands as in ALFF analysis. ReHo was then calculated for each sub-band. The spatial smoothing (FWHM $=6 \mathrm{~mm}$ ) was performed after ReHo calculation as did in previous studies (Zang et al., 2004).

\section{Calculation}

Degree centrality (DC) represents the node characteristic of large-scale brain intrinsic connectivity networks by capturing the relationship with the entire brain network in the voxel level (Zuo et al., 2012). We used weighted DC since it provides a more precise centrality characterization of functional brain networks than binary version (Cole et al., 2010). Specifically, after preprocessing, the linear trend of the time course of each voxel was removed, and then band-pass filtering was performed for six sub-bands as in ALFF analysis. The Pearson correlation was performed between the time course of each voxel with that of every other voxel in the entire brain (Buckner et al., 2009). The correlation coefficients with $r>0.2$ were summed up for each voxel and then a weighted DC was obtained for each voxel. 0.2 was used as threshold to eliminate counting voxels that had low temporal correlation and it has been proved that different threshold selections did not qualitatively change the results (Buckner et al., 2009). As did in ReHo calculation, spatial smoothing may introduce possible artificial local correlations, we performed spatial smoothing (FWHM $=6 \mathrm{~mm}$ ) after DC calculation as did elsewhere as follows (Zuo et al., 2012):

$$
\begin{aligned}
D & =\sum a_{i j} \\
\text { Where } j & =1 . . . N, i \neq j, a_{i j}=\left\{\begin{array}{c}
0, a_{i j}<0.2 \\
a_{i j}, a_{i j} \geqslant 0.2
\end{array}\right.
\end{aligned}
$$

Negative correlation was removed according to previous fMRI studies (Liao et al., 2013; Li et al., 2015). It was not calculated separately because the physiological basis of the negative correlations was ambiguous (Fox et al., 2009; Murphy et al., 2009).

ALFF measures the amplitude of time series fluctuation at each voxel (Zang et al., 2007), ReHo depicts the local synchronization of the time series of neighboring voxels (Zang 
et al., 2004), and DC represents the large-scale brain intrinsic connectivity in the voxel level (Buckner et al., 2009). Thus, the three measures of fMRI probe into the brain activity from different aspects.

\section{Statistical Analysis}

ALFF, ReHo, and DC maps of each frequency band were compared between the groups of children with ADHD and TDC. Two-sample $t$-tests were performed on the pooled data and each cohort, respectively. The full scale IQ and mean framewise displacement (FD) were included as nuisance covariates (Jenkinson et al., 2002; Yan et al., 2013), and cohort was further taken as a covariate for the $t$-tests on the pooled data. For each cohort, the statistical analyses were performed in study-specific functional volume masks including only voxels (in MNI152 standard space) present in at least $80 \%$ of the participants and then intersect with gray-matter mask to reduce non-cortical noise. The mask of the pooled data is the intersection of cohorts' masks. The results were corrected for multiple comparisons with a combined threshold of single voxel's $p<0.05$ and cluster size $>139,144,136,129$, and 129 voxels for the cohorts and pooled data, corresponding to corrected $p<$ 0.05 determined by Monte Carlo simulation and the mask of each cohort. The AlphaSim estimation was performed by DPABI V2.3 (http://rfmri.org/dpabi; Yan et al., 2016). At the same time, to reduce the possibility of false negative results and, hence, a more lenient threshold ( $p<0.05$, cluster size $>10$ voxels) was also used for each cohort.

We also performed the analyses of standardized effect size (SES) of each measurement based on Cohen's d which is calculated as the equation as follows (Cohen, 2013):

$$
\begin{aligned}
\text { Cohen's } d & =\frac{\bar{X}_{A D H D}-\bar{X}_{T D C}}{S_{A L L}}, \\
S_{A L L} & =\sqrt{\frac{\left(n_{A D H D}-1\right) S_{A D H D}^{2}+\left(n_{T D C}-1\right) S_{T D C}^{2}}{n_{A D H D}+n_{T D C}-2}}
\end{aligned}
$$

According to equation of independent two-sample $t$-test as follows:

$$
t=\frac{\bar{X}_{A D H D}-\bar{X}_{T D C}}{\sqrt{\frac{\left(n_{A D H D}-1\right) S_{A D H D}^{2}+\left(n_{T D C}-1\right) S_{T D C}^{2}}{n_{A D H D}+n_{T D C}-2}\left(\frac{1}{n_{A D H D}}+\frac{1}{n_{T D C}}\right)}}
$$

The relationship of Cohen's $d$ - and $t$-value can be obtained as follows:

$$
\text { Cohen's } d=t \sqrt{\frac{n_{A D H D}+n_{T D C}}{n_{A D H D} \cdot n_{T D C}}}
$$

According to Equation (5), we transformed t maps into SES map for each cohort and pooled data. Then a combined threshold SES $>0.30$ and cluster size $>129$ voxels was used which corresponded to a combination threshold of $t>1.974(p<0.05)$ of the pooled data. The same threshold was applied to the SES maps of each cohort. SES of 0.30 corresponded to $t=1.141$,
$0.822,1.124$, and 0.926 ( $p=0.26,0.43,0.27$, and 0.36 ) for NYU, PKU1, PKU2, and PKU3, respectively.

To view the consistency of results, the thresholded $t$-maps and SES-maps were binarized and overlapped among the four cohorts. Further, in order to view how consistent the results of individual cohorts are with the pooled results, the overlapped map of cohorts was further overlapped with the binary map of the pooled data. The number of overlapped voxels across 4 and 3 cohorts was quantified using Dice overlap coefficient (Dice, 1944; Burunat et al., 2016) where the voxel number of intersection was divided by the total voxel number of all the cohorts.

\section{RESULTS}

\section{Results of Pooled Data in Conventional Frequency Band}

The abnormal brain regions in the conventional low frequency band (0.01-0.08 Hz) for children with ADHD of the pooled data were shown in Figure 2 and Table 2. Children with ADHD had increased ALFF and DC in the bilateral lingual gyrus (Figures 2A,C). ReHo and DC were decreased in the bilateral cerebellum. In addition, the three methods detected some method-specific abnormality such as the bilateral paracentral lobule (Figure 2B) and the left insula (Figure 2C).

\section{Consistency across Cohorts in Conventional Frequency Band}

The abnormal brain activity in the conventional frequency band (0.01-0.08 Hz) was identified for each cohort, and the overlapped results across the four cohorts were shown in Figure 3 (See details of each cohort in Supplementary Figures 1-6). Only a few voxels showed overlapped abnormality from three or four cohorts by any method (ALFF, ReHo, or DC). Using DC, we observed 6 voxels overlapped from NYU, PKU2, and PKU3 in the left inferior occipital gyrus and fusiform gyrus. Even if taking the overlapped abnormality from 2 cohorts into consideration, only a few clusters were overlapped, e.g., the cerebellum by ReHo as well by DC (Figures 3B,C), the bilateral cuneus (Figure 3C) by DC.

The overlapped results of the pooled data and individual cohorts were shown in Figure 4. Some clusters detected in individual cohorts could not be observed in the results of pooled data, e.g., in the cuneus for ReHo (purple marked in Figure 4B) and thalamus for DC (purple marked in Figure 4C). Although, some clusters could be identified as the overlapped regions from two cohorts, they were not be observed in the pooled data, such as the right cerebellum for ReHo (yellow marked in Figure 4B).

The overlapped SES maps of each cohort and pooled data for ALFF, ReHo, and DC were shown in Figure 5 (with a combined threshold of SES > 0.3). Some clusters showing overlaps from more than 3 cohorts could be also shown in the pooled data (red marked). These clusters included the bilateral cerebellum for ReHo and DC (Figures 5B,C), right calcarine for ALFF and DC (Figures 5A,C) and the bilateral paracentral lobule for $\mathrm{ReHo}$ (Figure 5B). However, if the SES threshold was set at 0.5 , these 


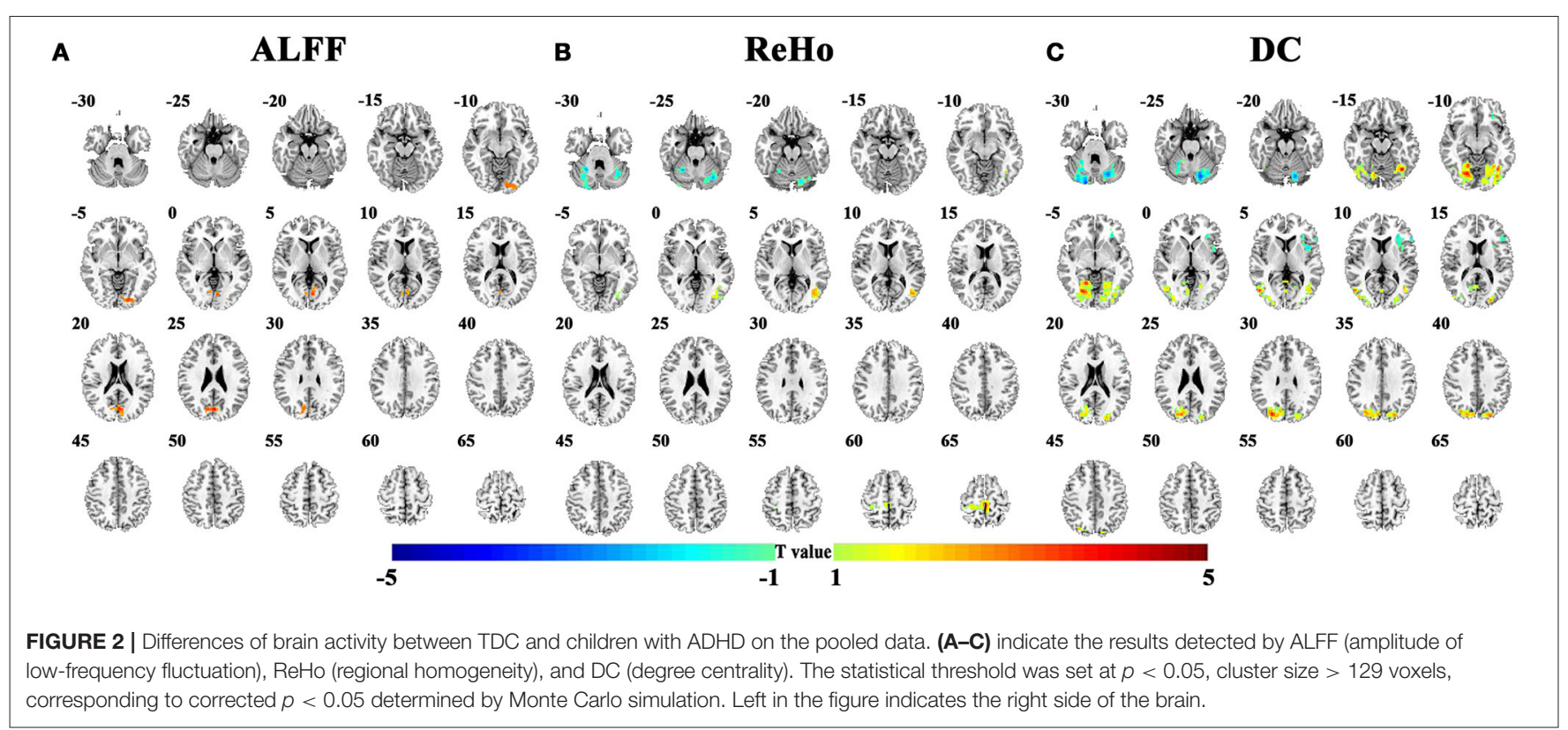

TABLE 2 | Differences between TDC and ADHD on pooled data.

\begin{tabular}{|c|c|c|c|c|c|c|c|c|}
\hline \multirow[t]{2}{*}{ Method } & \multirow[t]{2}{*}{ Region } & \multirow[t]{2}{*}{ L/R } & \multirow[t]{2}{*}{ BA } & \multicolumn{3}{|c|}{ Peak MNI coordinates } & \multirow[t]{2}{*}{$t$} & \multirow[t]{2}{*}{ Number of voxels } \\
\hline & & & & $x$ & $y$ & $z$ & & \\
\hline ALFF & Lingual gyrus/Cuneus & L/R & 18 & 0 & -75 & 24 & 3.59 & 236 \\
\hline \multirow[t]{3}{*}{$\mathrm{ReHo}$} & Cerebellum & $\mathrm{L} / \mathrm{R}$ & - & 33 & -69 & -36 & -3.70 & 537 \\
\hline & Paracentral lobule/Postcentral gyrus & $\mathrm{L} / \mathrm{R}$ & $3 / 4 / 6$ & -3 & -33 & 69 & 4.15 & 187 \\
\hline & Mid. temporal gyrus & $\mathrm{L}$ & 19 & -6 & 51 & -6 & 3.34 & 332 \\
\hline \multirow[t]{4}{*}{ DC } & Cerebellum & L/R & - & -18 & -75 & -24 & -3.96 & 744 \\
\hline & Mid. occipital/Lingual gyrus & $\mathrm{R}$ & 18 & 21 & -72 & -9 & 4.28 & 1,054 \\
\hline & Mid. occipital/Lingual gyrus & $\mathrm{L}$ & 18 & -39 & -63 & -12 & 4.30 & 635 \\
\hline & Insula & $\mathrm{L}$ & 13 & -30 & 30 & 9 & -3.15 & 202 \\
\hline
\end{tabular}

ALFF, amplitude of low-frequency fluctuation; ReHo, regional homogeneity; DC, degree centrality; Mid., middle; L, left; R, right; BA, Brodmann's area.

clusters showed no overlap (Figure 6). The overlapped SES maps across the 4 cohorts and the SES maps of the pooled data were shown in Supplementary Figure 7.

\section{Consistency across Cohorts in Sub-Frequency Bands}

After investigation in conventional low frequency band (0.01$0.08 \mathrm{~Hz}$ ) as shown above, overlapped results across cohort were further examined in several sub-frequency bands including Slow$6 / 5 / 4 / 3 / 2$. Furthermore, to reduce the possibility of false negative results and, a more lenient threshold $(p<0.05$, cluster size $>$ 10 voxels) was also applied for each cohort. There is no voxel overlapped by all the cohorts. The number of the overlapped voxels was not more than 12 across three cohorts, and the highest Dice overlap coefficient is only 0.0131 (Table 3). In each subfrequency band, most overlapped clusters were also observed from 2 cohorts (see details in Supplementary Figures 8-10).

\section{DISCUSSION}

The present study examined the consistency of abnormal local brain activity across cohorts of ADHD-200. We applied three voxel-wise whole brain analytic methods (ALFF, ReHo, and DC), strict and lenient statistical thresholds, and conventional frequency band (0.01-0.08 Hz) and sub-frequency bands (Slow/2/3/4/5/6) in the analysis process. Results from these analyses indicated that the abnormal local brain activity across cohorts of ADHD-200 was inconsistent.

The data of all four cohorts were first pooled together in the present study, as the general process way of the studies using ADHD-200 (Sato et al., 2013; Zhang et al., 2014). The abnormal brain activity for ADHD was identified in the clusters, such as the bilateral sensorimotor cortices and the bilateral lingual gyrus. Our further analysis showed that these results from pooled data were not consistent across cohorts. Most of the clusters identified for pooled data could not be observed in the results for individual 


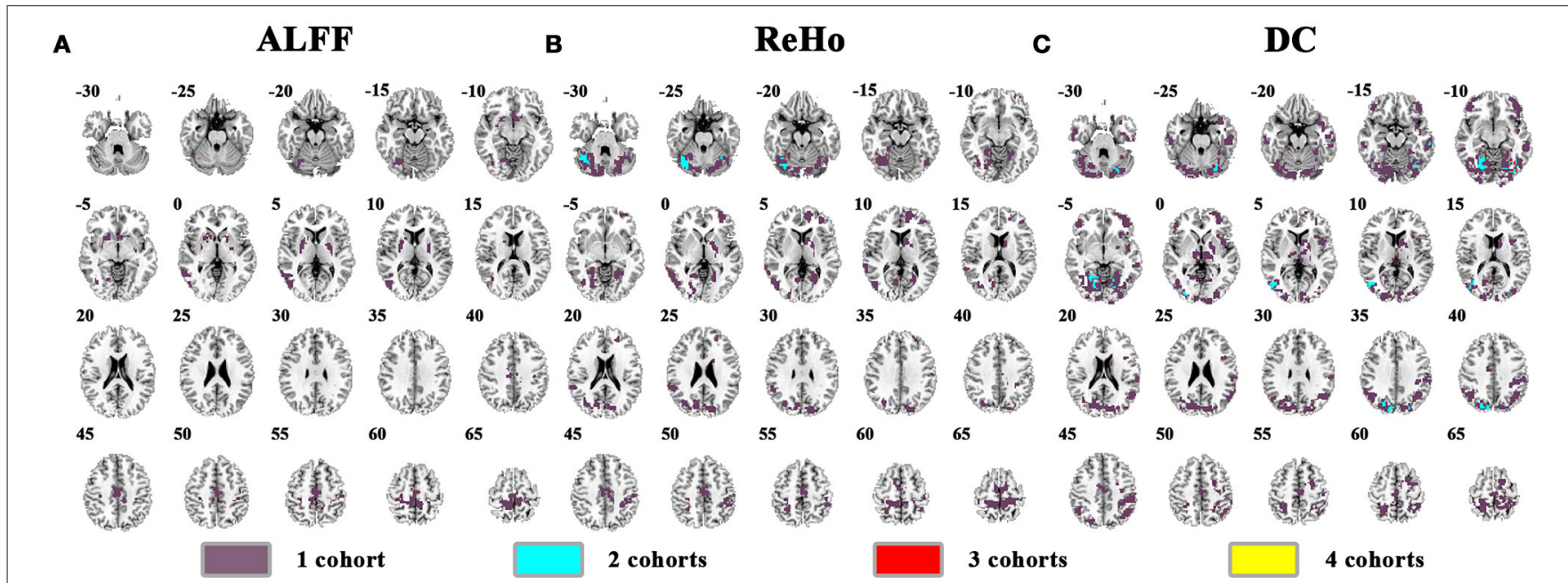

FIGURE 3 | The overlapped results across the 4 cohorts. (A-C) indicate the results detected by ALFF, ReHo, and DC, respectively. Purple indicates the regions detected in only one of the 4 cohorts. Mint, red, and yellow indicate the regions detected in 2, 3, and 4 cohorts, respectively.

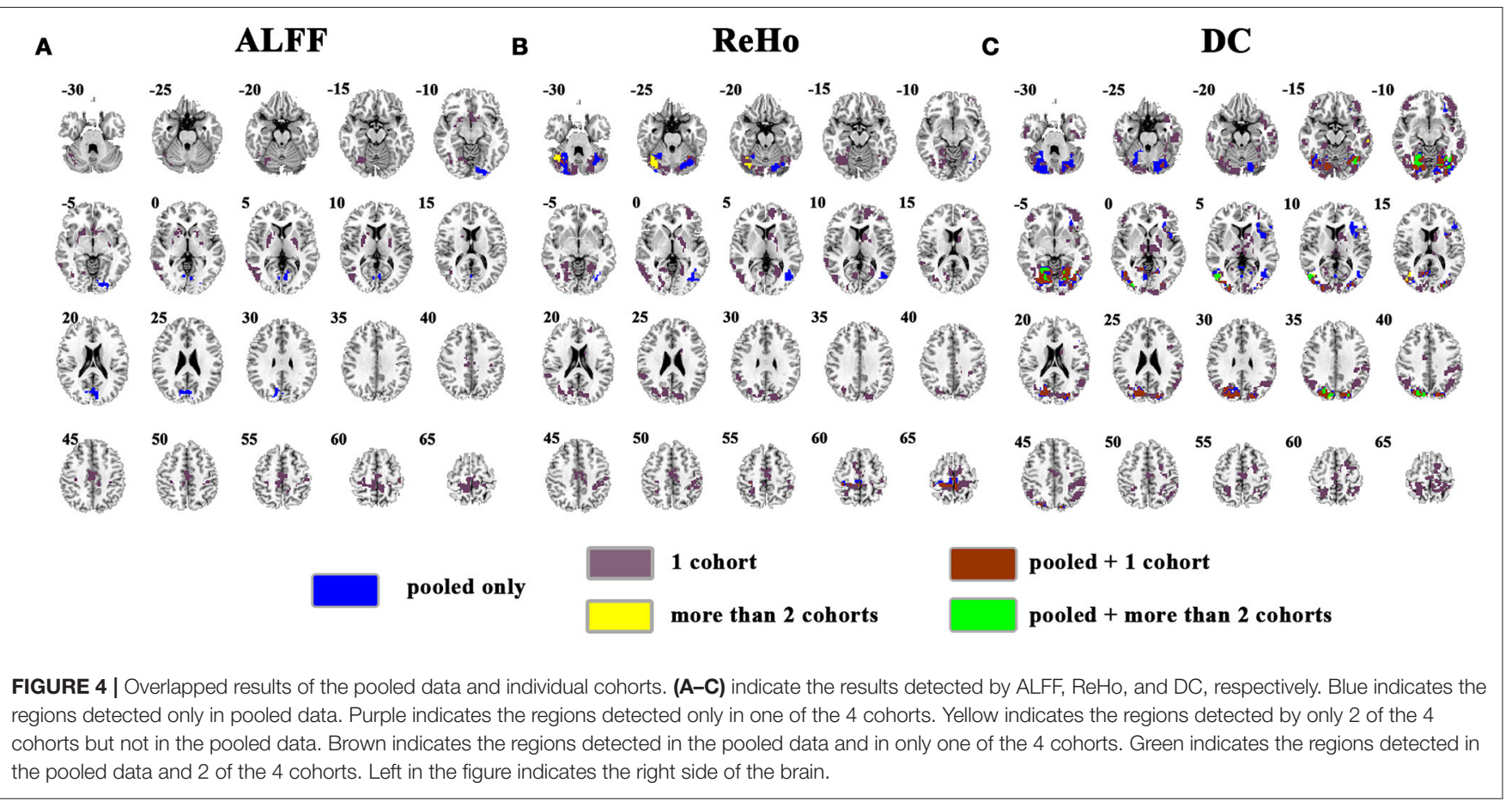

cohort. This finding was further supported by the analyses of SES. The overlapped regions did not reach a medium (0.5) level. Thus, the results of directly pooled data from different cohorts do not mean consistent results among the cohorts included, and the SES of the results should be examined in the future studies of large sample dataset. Future studies derived from multi-site largesample dataset should not only present the statistical result of a pooled data, but also present the results of each cohort of both $t$-map and SES.

Moreover, all examined cohorts did not exhibit overlapped clusters, suggesting a high heterogeneity of ADHD. We noticed a recent finding that detected the consistent abnormality across cohorts of ADHD-200 (Cai et al., 2015). Using the resource allocation index (RAI) (a measure of network interactions across the SN, CEN, and DMN), Cai et al. found RAI was significantly lower in children with ADHD than in control subjects and the results were reproducible across three independent cohorts. While abnormality of network interaction may reveal the complexity of spontaneous brain activity in ADHD, it could not illustrate which brain region is abnormal. From the perspective of clinical practice, analytic methods for precise localization of the abnormality in a whole-brain voxel-based 


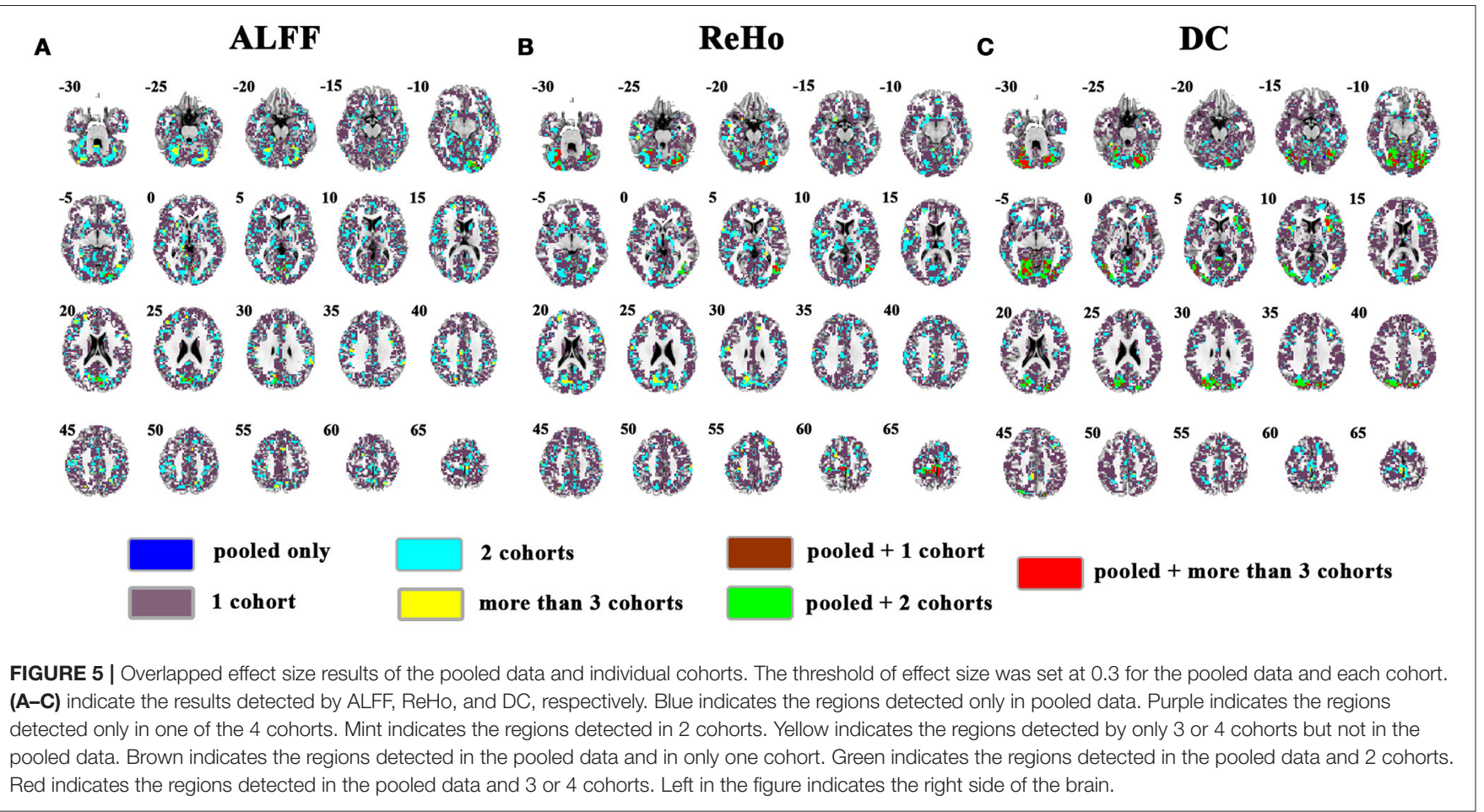

A

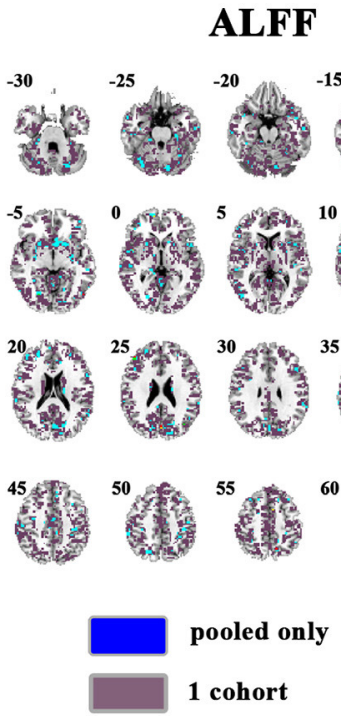

B
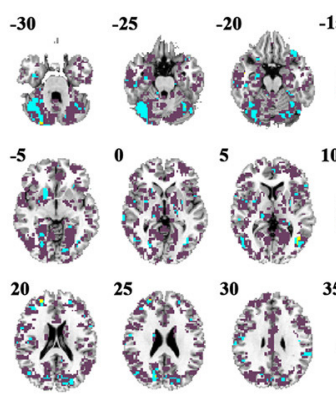

${ }^{20} x^{2}{ }^{2}$
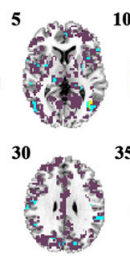
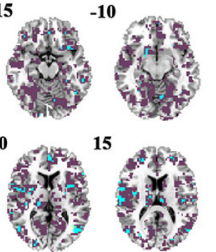

$\left.{ }^{35}\right)^{40}{ }^{4} y^{2}$
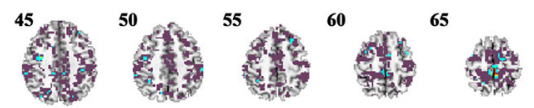

C

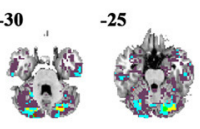

DC
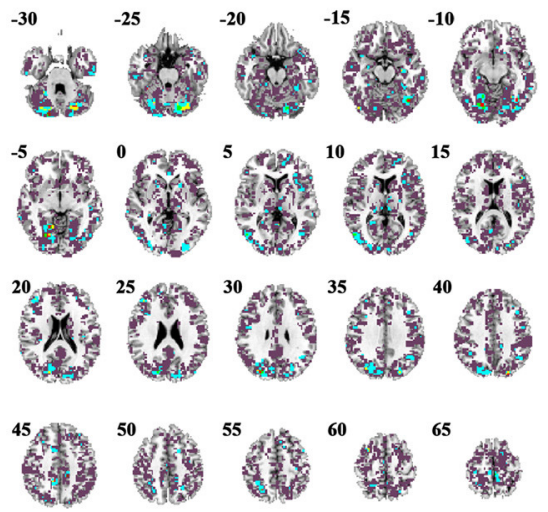

2 cohorts

more than 3 cohorts

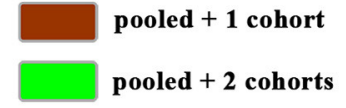

pooled + more than 3 cohorts

FIGURE 6 | Overlapped effect size results of the pooled data and individual cohorts. The threshold of effect size was set at 0.5 for the pooled data and each cohort. (A-C) indicate the results detected by ALFF, ReHo, and DC, respectively. Blue indicates the regions detected only in pooled data. Purple indicates the regions detected only in one of the 4 cohorts. Mint indicates the regions detected in 2 cohorts. Yellow indicates the regions detected by only 3 or 4 cohorts but not in the pooled data. Brown indicates the regions detected in the pooled data and in only one cohort. Green indicates the regions detected in the pooled data and 2 cohorts. Red indicates the regions detected in the pooled data and 3 or 4 cohorts. Left in the figure indicates the right side of the brain.

way should be emphasized. Whole-brain voxel-based analysis facilities coordinate-based meta-analysis (CB-meta) which can help to define precise localization of abnormal spontaneous brain activity by quantitatively aggregating independent results reported in a standard coordinate space (Eickhoff et al., 2009) and further help to guide intervention therapies, such as deep brain stimulation and transcranial magnetic stimulation (Zang et al., 2015). Thus, the present study used three whole-brain voxel-based measurements, i.e., ALFF, ReHo, and DC. These measurements are widely employed in rs-fMRI studies to access 
TABLE 3 | Clusters which were the overlap for three/four cohorts and contained maximal number of voxels.

\begin{tabular}{|c|c|c|c|c|c|c|}
\hline Method & $\begin{array}{c}\text { Number of } \\
\text { overlapped } \\
\text { cohorts }\end{array}$ & Region & L/R & BA & $\begin{array}{l}\text { Number of } \\
\text { overlapped } \\
\text { voxels }\end{array}$ & Dice \\
\hline \multicolumn{7}{|c|}{ CONVENTIONAL BAND $(0.01-0.08 \mathrm{~Hz})$} \\
\hline \multirow[t]{2}{*}{ ALFF } & 4 & & & None & & \\
\hline & 3 & & & None & & \\
\hline \multirow[t]{2}{*}{$\mathrm{ReHo}$} & 4 & & & None & & \\
\hline & 3 & $\begin{array}{l}\text { Paracentral } \\
\text { lobule }\end{array}$ & $\mathrm{R}$ & 4 & 12 & 0.0041 \\
\hline \multirow[t]{2}{*}{ DC } & 4 & & & None & & \\
\hline & 3 & Cerebellum & $\mathrm{R}$ & - & 12 & 0.0096 \\
\hline \multicolumn{7}{|c|}{ SLOW-6 (0-0.01 Hz) } \\
\hline \multirow[t]{2}{*}{ ALFF } & 4 & & & None & & \\
\hline & 3 & & & None & & \\
\hline \multirow[t]{2}{*}{$\mathrm{ReHo}$} & 4 & & & None & & \\
\hline & 3 & $\begin{array}{l}\text { Med. frontal } \\
\text { cortex }\end{array}$ & $\mathrm{L}$ & 11 & 1 & 0.0004 \\
\hline \multirow[t]{2}{*}{ DC } & 4 & & & None & & \\
\hline & 3 & & & None & & \\
\hline \multicolumn{7}{|c|}{ SLOW-5 (0.01-0.027 Hz) } \\
\hline \multirow[t]{2}{*}{ ALFF } & 4 & & & None & & \\
\hline & 3 & & & None & & \\
\hline \multirow[t]{2}{*}{$\mathrm{ReHo}$} & 4 & & & None & & \\
\hline & 3 & $\begin{array}{l}\text { Paracentral } \\
\text { lobule }\end{array}$ & L/R & 4 & 3 & 0.0016 \\
\hline \multirow[t]{2}{*}{ DC } & 4 & & & None & & \\
\hline & 3 & $\begin{array}{l}\text { Paracentral } \\
\text { lobule }\end{array}$ & L/R & 4 & 7 & 0.0025 \\
\hline
\end{tabular}

\begin{tabular}{|c|c|c|c|c|c|c|}
\hline \multicolumn{7}{|c|}{ SLOW-4 (0.027-0.073 Hz) } \\
\hline \multirow[t]{2}{*}{ ALFF } & 4 & & & None & & \\
\hline & 3 & & & None & & \\
\hline \multirow[t]{2}{*}{$\mathrm{ReHo}$} & 4 & & & None & & \\
\hline & 3 & Cerebellum & $\mathrm{R}$ & - & 6 & 0.0026 \\
\hline \multirow[t]{2}{*}{ DC } & 4 & & & None & & \\
\hline & 3 & $\begin{array}{l}\text { Mid. occipital } \\
\text { gyrus }\end{array}$ & $\mathrm{L}$ & 19 & 11 & 0.0131 \\
\hline \multicolumn{7}{|c|}{ SLOW-3 (0.073-0.198 Hz) } \\
\hline \multirow[t]{2}{*}{ ALFF } & 4 & & & None & & \\
\hline & 3 & $\begin{array}{l}\text { Paracentral } \\
\text { lobule }\end{array}$ & $\mathrm{L}$ & 4 & 1 & 0.0005 \\
\hline \multirow[t]{2}{*}{ ReHo } & 4 & & & None & & \\
\hline & 3 & $\begin{array}{l}\text { Mid. frontal } \\
\text { gyrus }\end{array}$ & $\mathrm{L}$ & 8 & 2 & 0.0013 \\
\hline \multirow[t]{2}{*}{ DC } & 4 & & & None & & \\
\hline & 3 & $\begin{array}{l}\text { Supplementary } \\
\text { motor area }\end{array}$ & R & 6 & 1 & 0.0004 \\
\hline \multicolumn{7}{|c|}{ SLOW-2 (0.198-0.25 Hz) } \\
\hline \multirow[t]{2}{*}{ ALFF } & 4 & & & None & & \\
\hline & 3 & & & None & & \\
\hline \multirow[t]{2}{*}{$\mathrm{ReHo}$} & 4 & & & None & & \\
\hline & 3 & $\begin{array}{l}\text { Sup. frontal } \\
\text { gyrus }\end{array}$ & $\mathrm{R}$ & 6 & 2 & 0.001 \\
\hline \multirow[t]{2}{*}{ DC } & 4 & & & None & & \\
\hline & 3 & & & None & & \\
\hline
\end{tabular}

Sup., superior; Mid., Middle; Med., Medial; L, left; R, right. The threshold was $p<0.05$ and cluster size $>10$ voxels for each cohort. local brain activity from different aspects. Here we applied these three measurements to explore the consistent local abnormality of children with ADHD across cohorts. Nevertheless, consistent results across cohorts were not identified through any one of the three measurements.

The present study not only focused on the conventional frequency band but also stressed several sub-frequency bands. Frequency-dependent investigation provides us a new prospect to investigate the physiological mechanism of the brain activity. A recent rs-fMRI study reported some frequency-dependent abnormalities for children with ADHD (Yu et al., 2015). For example, in the orbital frontal cortex (OFC), the frequency bands of slow-3 and slow-2 contributed more to the differences than did the slow- 5 and slow- 4 bands. We found that the detected differences between ADHD and TDC are different according to different frequency bands. For example, compared with TDC, children with ADHD had decreased DC in the left inferior parietal gyrus only in slow-3 but others frequency bands and decreased DC in the bilateral putamen/thalamus only in slow-4 but others frequency bands (Supplementary Figure 11). Previous studies often consider the Slow-6 $(<0.01 \mathrm{~Hz})$ as signal drift, and it was usually discarded from further analysis. However, our recent publications on finger force feedback task have challenged this issue. ReHo and ALFF of basal ganglia in Slow-6 showed difference between real and sham feedback conditions, and the ALFF in Slow-6 was related to finger force (Zhang et al., 2015a,b). Moreover, ReHo difference between ADHD and TDC in Slow-6 was detected in previous study (Yu et al., 2015). Thus, the Slow6 was involved in our analysis and differences can be detected. However, the results couldn't be detected in any other cohorts.

Several limitations exist in the present study. First, we could not explore the contribution of different subtype to the inconsistency in ADHD neuroimaging findings because of the small sample size for statistical analysis. For example, PKU1 only included 6 inattention and 9 combined subjects. Their contribution should be explored on a large sample dataset in the future. Second, we only used three whole brain voxelbased measurements to evaluate the consistency across cohorts. Thus, our observations were restricted to these measurements. Investigations with more whole-brain voxel-based measurements will be helpful.

\section{CONCLUSIONS}

Data-sharing projects like ADHD-200 provide large sample analysis. But pooled data itself is not enough. The current study used three whole-brain voxel-based analytic methods, i.e., ALFF, ReHo, and DC not only on the pooled data but also on each individual cohort. We found that the findings based on the pooled data of ADHD-200 were inconsistent across the individual cohorts. Even in a more lenient threshold, this inconsistency could be observed. Such inconsistency could be found not only in the conventional low frequency-band (0.01-0.08 Hz) but also in a few sub-frequency band of Slow$2 / 3 / 4 / 5 / 6$. These results support the view that ADHD is a highly heterogeneous disorder. Future studies should try more efforts 
on exploring more consistent findings of rs-fMRI data of ADHD. Data sharing could benefit improving the reproducibility of neuroimage studies, and we suggest that analysis based on multisite large-sample dataset should be performed on pooled data and single cohort, respectively.

\section{AUTHOR CONTRIBUTIONS}

YZ, HZ, and QC conceived and designed the experiment. JW and $\mathrm{HZ}$ performed the data analysis. YZ, LZ, LS, and YW provided advice on the analysis and interpretation of the results. JW, HZ, and $\mathrm{YZ}$ wrote the paper.

\section{FUNDING}

This work is supported by grants from the National Key Basic Research Program of China [973 Program, 2014CB846104], the National Natural Science Foundation of China [81271652,

\section{REFERENCES}

ADHD-200-Consortium, T. (2012). The ADHD-200 consortium: a model to advance the translational potential of neuroimaging in clinical neuroscience. Front. Syst. Neurosci. 6:62. doi: 10.3389/fnsys.2012.00062

Bartra, O., McGuire, J. T., and Kable, J. W. (2013). The valuation system: a coordinate-based meta-analysis of BOLD fMRI experiments examining neural correlates of subjective value. Neuroimage 76, 412-427. doi: 10.1016/j.neuroimage.2013.02.063

Buckner, R. L., Sepulcre, J., Talukdar, T., Krienen, F. M., Liu, H., Hedden, T., et al. (2009). Cortical hubs revealed by intrinsic functional connectivity: mapping, assessment of stability, and relation to Alzheimer's disease. J. Neurosci. 29, 1860-1873. doi: 10.1523/JNEUROSCI.5062-08.2009

Burunat, I., Toiviainen, P., Alluri, V., Bogert, B., Ristaniemi, T., Sams, M., et al. (2016). The reliability of continuous brain responses during naturalistic listening to music. Neuroimage 124, 224-231. doi: 10.1016/j.neuroimage.2015.09.005

Bush, G. (2010). Attention-deficit/hyperactivity disorder and attention networks. Neuropsychopharmacology 35, 278-300. doi: 10.1038/npp.2009.120

Cai, W., Chen, T., Szegletes, L., Supekar, K., and Menon, V. (2015). Aberrant cross-brain network interaction in children with attentiondeficit/hyperactivity disorder and its relation to attention deficits: a multisite and cross-site replication study. Biol. Psychiatry. doi: 10.1016/j.biopsych.2015. 10.017. [Epub ahead of print].

Carmona, S., Hoekzema, E., Castellanos, F. X., Garcia-Garcia, D., LageCastellanos, A., Van Dijk, K. R., et al. (2015). Sensation-to-cognition cortical streams in attention-deficit/hyperactivity disorder. Hum. Brain Mapp. 36, 2544-2557 doi: 10.1002/hbm.22790

Castellanos, F. X., Sonuga-Barke, E. J. S., Milham, M. P., and Tannock, R. (2006). Characterizing cognition in ADHD: beyond executive dysfunction. Trends Cogn. Sci. 10, 117-123. doi: 10.1016/j.tics.2006.01.011

Chao-Gan, Y., and Yu-Feng, Z. (2010). DPARSF: a MATLAB toolbox for "pipeline" data analysis of resting-state fMRI. Front. Syst. Neurosci. 4:13. doi: 10.3389/fnsys. 2010.00013

Cohen, J. (2013). Statistical Power Analysis for the Behavioral Sciences. New York, NY: Routledge.

Cole, M. W., Pathak, S., and Schneider, W. (2010). Identifying the brain's most globally connected regions. Neuroimage 49, 3132-3148. doi: 10.1016/j.neuroimage.2009.11.001

Cortese, S., Kelly, C., Chabernaud, C., Proal, E., Di Martino, A., Milham, M. P., et al. (2012). Toward systems neuroscience of ADHD: a meta-analysis of 55 fMRI studies. Am. J. Psychiatry 169, 1038-1055. doi: 10.1176/appi.ajp.2012.11101521
81520108016, 31471084, 81401481, 81371496, and 81471382], the Open Research Fund of the State Key Laboratory of Cognitive Neuroscience and Learning [CNLYB1508] and Key Laboratory for Magnetic Resonance and Multimodality Imaging of Guangdong Province [2014B030301013]. YZ is partly supported by "Qian Jiang Distinguished Professor" program.

\section{ACKNOWLEDGMENTS}

The authors acknowledge the contribution of ADHD-200 consortium organizers for sharing the raw data.

\section{SUPPLEMENTARY MATERIAL}

The Supplementary Material for this article can be found online at: http://journal.frontiersin.org/article/10.3389/fnins. 2017.00320/full\#supplementary-material

Dice, L. R. (1944). Measures of the amount of ecologic association between species. Ecology 26, 297-302. doi: 10.2307/1932409

Eickhoff, S. B., Laird, A. R., Grefkes, C., Wang, L. E., Zilles, K., and Fox, P. T. (2009). Coordinate-based activation likelihood estimation meta-analysis of neuroimaging data: a random-effects approach based on empirical estimates of spatial uncertainty. Hum. Brain Mapp. 30, 2907-2926. doi: 10.1002/hbm. 20718

Elton, A., Alcauter, S., and Gao, W. (2014). Network connectivity abnormality profile supports a categorical-dimensional hybrid model of ADHD. Hum. Brain Mapp. 35, 4531-4543. doi: 10.1002/hbm.22492

Fox, M. D., Zhang D., Snyder, A. Z., and Raichle, M. E. (2009). The global signal and observed anticorrelated resting state brain networks. J. Neurophysiol. 101, 3270-3283. doi: 10.1152/jn.90777.2008

Han, Y., Wang, J., Zhao, Z., Min, B., Lu, J., Li, K., et al. (2011). Frequencydependent changes in the amplitude of low-frequency fluctuations in amnestic mild cognitive impairment: a resting-state fMRI study. Neuroimage 55, 287-295. doi: 10.1016/j.neuroimage.2010.11.059

Herz, D. M., Eickhoff, S. B., Lokkegaard, A., and Siebner, H. R. (2014). Functional neuroimaging of motor control in Parkinson's disease: a meta-analysis. Hum. Brain Mapp. 35, 3227-3237. doi: 10.1002/hbm.22397

Iwabuchi, S. J., Krishnadas, R., Li, C., Auer, D. P., Radua, J., and Palaniyappan, L. (2015). Localized connectivity in depression: a meta-analysis of resting state functional imaging studies. Neurosci. Biobehav. Rev. 51, 77-86. doi: 10.1016/j.neubiorev.2015.01.006

Jenkinson, M., Bannister, P., Brady, M., and Smith, S. (2002). Improved optimization for the robust and accurate linear registration and motion correction of brain images. Neuroimage 17, 825-841. doi: 10.1006/nimg.2002.1132

Li, S., Ma, X., Huang, R., Li, M., Tian, J., Wen, H., et al. (2015). Abnormal degree centrality in neurologically asymptomatic patients with end-stage renal disease: a resting-state fMRI study. Clin Neurophysiol. 127, 602-609. doi: 10.1016/j.clinph.2015.06.022

Liao, X.-H., Xia, M.-R., Xu, T., Dai, Z.-J., Cao, X.-Y., Niu, H.-J., et al. (2013). Functional brain hubs and their test-retest reliability: a multiband resting-state functional MRI study. Neuroimage 83, 969-982. doi: 10.1016/j.neuroimage.2013.07.058

Lv, Y., Margulies, D. S., Villringer, A., and Zang, Y.-F. (2013). Effects of finger tapping frequency on regional homogeneity of sensorimotor cortex. PLoS ONE 8:e64115. doi: 10.1371/journal.pone.0064115

Malinen, S., Vartiainen, N., Hlushchuk, Y., Koskinen, M., Ramkumar, P., Forss, N., et al. (2010). Aberrant temporal and spatial brain activity during rest in patients with chronic pain. Proc. Natl. Acad. Sci. U.S.A. 107, 6493-6497. doi: $10.1073 /$ pnas. 1001504107 
Mills, K. L., Bathula, D., Dias, T. G., Iyer, S. P., Fenesy, M. C., Musser, E. D., et al. (2012). Altered cortico-striatal-thalamic connectivity in relation to spatial working memory capacity in children with ADHD. Front. Psychiatry 3:2. doi: 10.3389/fpsyt.2012.00002

Molloy, E. K., Meyerand, M. E., and Birn, R. M. (2014). The influence of spatial resolution and smoothing on the detectability of resting-state and task fMRI. Neuroimage 86, 221-230. doi: 10.1016/j.neuroimage.2013.09.001

Murphy, K., Birn, R. M., Handwerker, D. A., Jones, T. B., and Bandettini, P. A. (2009). The impact of global signal regression on resting state correlations: are anti-correlated networks introduced? Neuroimage 44, 893-905. doi: 10.1016/j.neuroimage.2008.09.036

Newman, E., Jernigan, T. L., Lisdahl, K. M., Tamm, L., Tapert, S. F., Potkin, S. G., et al. (2015). Go/No Go task performance predicts cortical thickness in the caudal inferior frontal gyrus in young adults with and without ADHD. Brain Imaging Behav. 10, 880-892. doi: 10.1007/s11682-015-9453-x

Otti, A., Guendel, H., Wohlschlager, A., Zimmer, C., and Noll-Hussong, M. (2013). Frequency shifts in the anterior default mode network and the salience network in chronic pain disorder. BMC Psychiatry 13:84. doi: 10.1186/1471-244X-13-84

Polanczyk, G. V., Salum, G. A., Sugaya, L. S., Caye, A., and Rohde, L. A. (2015). Annual research review: a meta-analysis of the worldwide prevalence of mental disorders in children and adolescents. J. Child Psychol. Psychiatry 56, 345-365. doi: $10.1111 /$ jcpp. 12381

Sato, J. R., Takahashi, D. Y., Hoexter, M. Q., Massirer, K. B., and Fujita, A. (2013). Measuring network's entropy in ADHD: a new approach to investigate neuropsychiatric disorders. Neuroimage 77, 44-51. doi: 10.1016/j.neuroimage.2013.03.035

Schulz, K. P., Fan, J., Tang, C. Y., Newcorn, J. H., Buchsbaum, M. S., Cheung, A. M., et al. (2004). Response inhibition in adolescents diagnosed with attention deficit hyperactivity disorder during childhood: an event-related fMRI study. Am. J. Psychiatry 161, 1650-1657. doi: 10.1176/appi.ajp.161.9.1650

Song, X. W., Dong, Z. Y., Long, X. Y., Li, S. F., Zuo, X. N., Zhu, C. Z., et al. (2011). REST: a toolkit for resting-state functional magnetic resonance imaging data processing. PLoS ONE 6:e25031. doi: 10.1371/journal.pone.0025031

Sripada, C., Kessler, D., Fang, Y., Welsh, R. C., Prem Kumar, K., and Angstadt, M. (2014). Disrupted network architecture of the resting brain in attention-deficit/hyperactivity disorder. Hum. Brain Mapp. 35, 4693-4705. doi: $10.1002 / \mathrm{hbm} .22504$

Tomasi, D., and Volkow, N. D. (2012). Abnormal functional connectivity in children with attention-deficit/hyperactivity disorder. Biol. Psychiatry 71, 443-450. doi: 10.1016/j.biopsych.2011.11.003

Vaidya, C. J., Bunge, S. A., Dudukovic, N. M., Zalecki, C. A., Elliott, G. R., and Gabrieli, J. D. E. (2005). Altered neural substrates of cognitive control in childhood ADHD: evidence from functional magnetic resonance imaging. Am. J. Psychiatry 162, 1605-1613. doi: 10.1176/appi.ajp.162.9.1605

Vasic, N., Plichta, M. M., Wolf, R. C., Fallgatter, A. J., Sosic-Vasic, Z., and Gron, G. (2014). Reduced neural error signaling in left inferior prefrontal cortex in young adults with ADHD. J. Atten. Disord. 18, 659-670. doi: $10.1177 / 1087054712446172$

Wang, J., Zhang, Z., Ji, G. J., Xu, Q., Huang, Y., Wang, Z., et al. (2015). Frequencyspecific alterations of local synchronization in idiopathic generalized epilepsy. Medicine 94:e1374. doi: 10.1097/md.0000000000001374

Yan, C. G., Cheung, B., Kelly, C., Colcombe, S., Craddock, R. C., Di Martino, A., et al. (2013). A comprehensive assessment of regional variation in the impact of head micromovements on functional connectomics. Neuroimage 76, 183-201. doi: 10.1016/j.neuroimage.2013.03.004
Yan, C. G., Wang, X. D., Zuo, X. N., and Zang, Y. F. (2016). DPABI: data processing \& analysis for (resting-state) brain imaging. 14, 339-351. doi: 10.1007/s12021-016-9299-4

Yu, X., Yuan, B., Cao, Q., An, L., Wang, P., Vance, A., et al. (2015). Frequencyspecific abnormalities in regional homogeneity among children with attention deficit hyperactivity disorder: a resting-state fMRI study. Sci. Bull. 61, 682-692. doi: 10.1007/s11434-015-0823-y

Yuan, B. K., Wang, J., Zang, Y. F., and Liu, D. Q. (2014). Amplitude differences in high-frequency fMRI signals between eyes open and eyes closed resting states. Front. Hum. Neurosci. 8:503. doi: 10.3389/fnhum.2014. 00503

Zang, Y., Jiang, T., Lu, Y., He, Y., and Tian, L. (2004). Regional homogeneity approach to fMRI data analysis. Neuroimage 22, 394-400. doi: 10.1016/j.neuroimage.2003.12.030

Zang, Y. F., He, Y., Zhu, C. Z., Cao, Q. J., Sui, M. Q., Liang, M., et al. (2007). Altered baseline brain activity in children with ADHD revealed by restingstate functional MRI. Brain Dev. 29, 83-91. doi: 10.1016/j.braindev.2006. 07.002

Zang, Y. F., Zuo, X. N., Milham, M., and Hallett, M. (2015). Toward a meta-analytic synthesis of the resting-state fMRI literature for clinical populations. Biomed Res. Int. 2015:435265. doi: 10.1155/2015/435265

Zhang, H., Gao, Z. Z., and Zang, Y. F. (2015a). An fMRI study of local synchronization in different subfrequency bands during the continuous feedback of finger force. Biomed Res. Int. 2015:273126. doi: $10.1155 / 2015 / 273126$

Zhang, H., Zhang, L., and Zang, Y. (2015b). Fluctuation amplitude and local synchronization of brain activity in the ultra-low frequency band: an fMRI investigation of continuous feedback of finger force. Brain Res. 1629, 104-112. doi: 10.1016/j.brainres.2015.10.023

Zhang, J., Kendrick, K. M., Lu, G., and Feng, J. (2014). The fault lies on the other side: altered brain functional connectivity in psychiatric disorders is mainly caused by counterpart regions in the opposite hemisphere. Cereb. Cortex 25 , 3475-3486. doi: 10.1093/cercor/bhu173

Zhang, J., Wei, L., Hu, X., Zhang, Y., Zhou, D., Li, C., et al. (2013). Specific frequency band of amplitude low-frequency fluctuation predicts Parkinson's disease. Behav. Brain Res. 252, 18-23. doi: 10.1016/j.bbr.2013. 05.039

Zuo, X.-N., Di Martino, A., Kelly, C., Shehzad, Z. E., Gee, D. G., Klein, D. F., et al. (2010). The oscillating brain: complex and reliable. Neuroimage 49, 1432-1445. doi: 10.1016/j.neuroimage.2009.09.037

Zuo, X. N., Ehmke, R., Mennes, M., Imperati, D., Castellanos, F. X., Sporns, O., et al. (2012). Network centrality in the human functional connectome. Cereb. Cortex 22, 1862-1875. doi: 10.1093/cercor/bhr269

Conflict of Interest Statement: The authors declare that the research was conducted in the absence of any commercial or financial relationships that could be construed as a potential conflict of interest.

Copyright (c) 2017 Wang, Zheng, Cao, Wang, Sun, Zang and Zhang. This is an open-access article distributed under the terms of the Creative Commons Attribution License (CC BY). The use, distribution or reproduction in other forums is permitted, provided the original author(s) or licensor are credited and that the original publication in this journal is cited, in accordance with accepted academic practice. No use, distribution or reproduction is permitted which does not comply with these terms. 